\title{
Design and Implementation of the Phase-locked Amplifier
}

\author{
Mingxin Song and Fangfang Liu \\ Harbin University of Science and Technology \\ songmingxin@126.com
}

\begin{abstract}
The phase-locked amplifier with output stability can filter out the noise effectively and the weak signal will be extracted from the noise and be amplified. The signal processing is relatively simple, so it is an effective way to detect the weak signal. It uses the phase sensitive detector (Phase Sensitive Detection, PSD) technique to identify the test signal, which has same frequency with the reference signal, and eliminate the interference noise signal of different frequency, so as to accurately measure the size of the measured signal. In this paper, switching correlator is taken as a core part of lock-in amplifier with large dynamic range, strong anti-overload and no non-linear problems. Moreover, lock-in amplifier output signal amplitude has no impact from the amplitude of the reference.
\end{abstract}

Keywords: phase-locked amplifier, PSD, noise

\section{Introduction}

When the weak optical signal is detected, because of the presence of background noise and other interference noise, the signal is usually buried in the noise, so it not only brings a great error to detection, but also reduces the dynamic range of the detection system. Measurement technology needs development and improvement in two aspects, speed and accuracy. The phase-locked amplifier as an effective method for accurate detection of weak signals on these two issues can achieve high demand with significant advantages. Lock-in amplifier (Lock- in Amplified LLl), as the selected frequency amplifier, not only uses the frequency characteristics of the signal, but also captures the phase characteristics of the signal, namely, "lock" the phase of the measured signal. It has an important role in weak signal measurement, and it has attracted more and more attention [1].

\section{Principles of Week Signal Detection}

Phase-locked amplifier for signal spectrum migration process is shown in Figure 1. The low-frequency signal is multiplied by the frequency modulation process w0 sinusoidal carrier, and the migration of the spectrum to the modulation frequency is on either side of the w0 after frequency selective amplifier, so as not to put the $1 / \mathrm{f}$ noise and low frequency drift is magnified, as shown in Figure 1 (a). The dotted line shows the density of $1 / \mathrm{f}$ noise and white noise power spectral. After they are ac amplified with phase sensitive detector (PSD) in spectrum moved to DC ( $\omega=0)$ on both sides, narrow band low pass filter (LPF) filters out noise to get the high signal-to-noise ratio of signal to enlarge, as shown in Figure 1 (b). The dotted line represents LPF frequency response curve. As long as the bandwidth of the LPF is enough, it can effectively improve the signal-to-noise ratio [2]. 

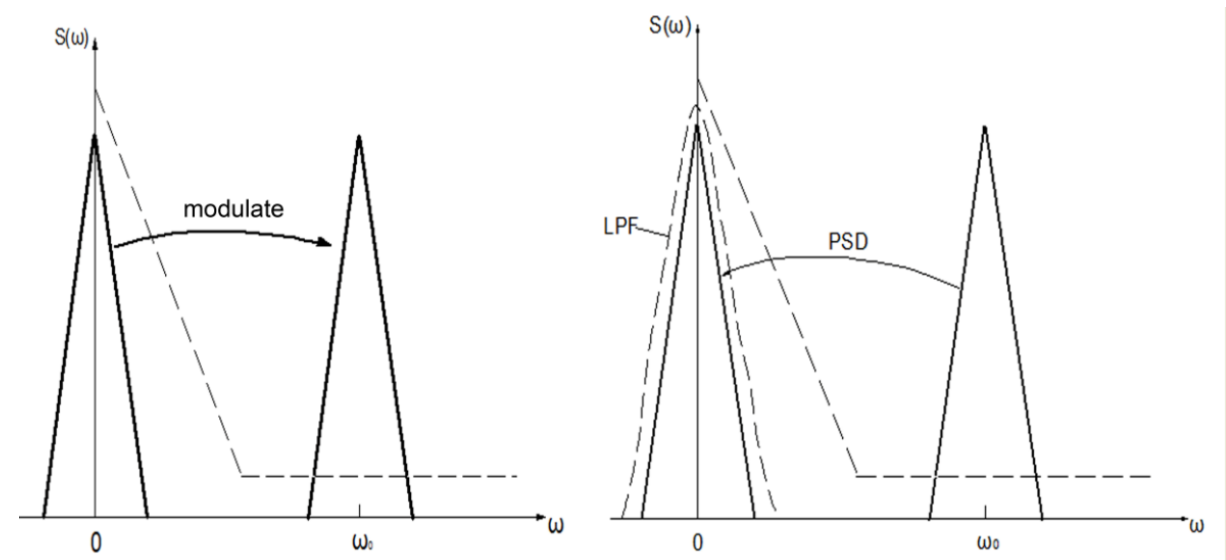

(a) Modulation Process; (b) Phase Sensitive Detection Process

\section{Figure 1. The Frequency Spectrum of the Phase-locked Amplifier Migration Process}

\section{The Overall Design of Phase-locked Amplifier}

The basic structure of the phase-locked amplifier is shown in Figure 2, including signal channel, reference channel, phase sensitive detector (PSD) and the low pass filter (LPF) [3]. Signal channel communicates modulated sine signal amplification and amplifies weak signal to make it strong enough to move the phase-sensitive detector work level, and to filter out some interference and noise and consequently to improve the dynamic range of phase-sensitive detector. The reference input is usually continuous sinusoidal signals or square wave switch, it can be a periodic signal from external input, also can be in the original system used for modulation of the carrier signal or used for chopping signal. Reference channel attenuation enlarges the reference input or adapts the demand of phase-sensitive detector for amplitude. Reference channel is another important feature of the reference input phase shift processing, making all sorts of different phase shift signal detection to achieve the best results.

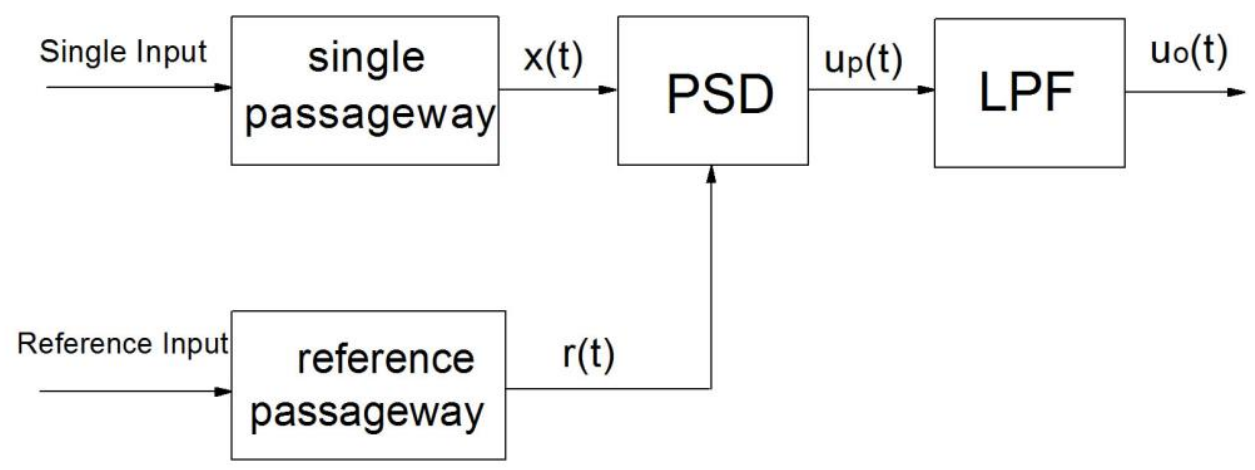

Figure 2. Working Principle of the Phase-locked Amplifier

Lock-in amplifier is mainly composed of the signal path, the reference channel and the phase-sensitive detector. The signal channel, in order to pre-process the input signal, is weak. Weak signal is amplified to a level sufficient to promote the subsequent stage; reference channel is a reference signal pre-processing part with whole type, phase shift reference signal appropriate for amplification, making the reference signal into a 50\% 
duty cycle square wave, as the tracking bandpass filter and phase sensitive detector switching signal. Phase sensitive detector is the core part of the whole system, it treats the input signal and the reference signal corresponding operation, and low pass filter. The output signal is proportional to a DC input.

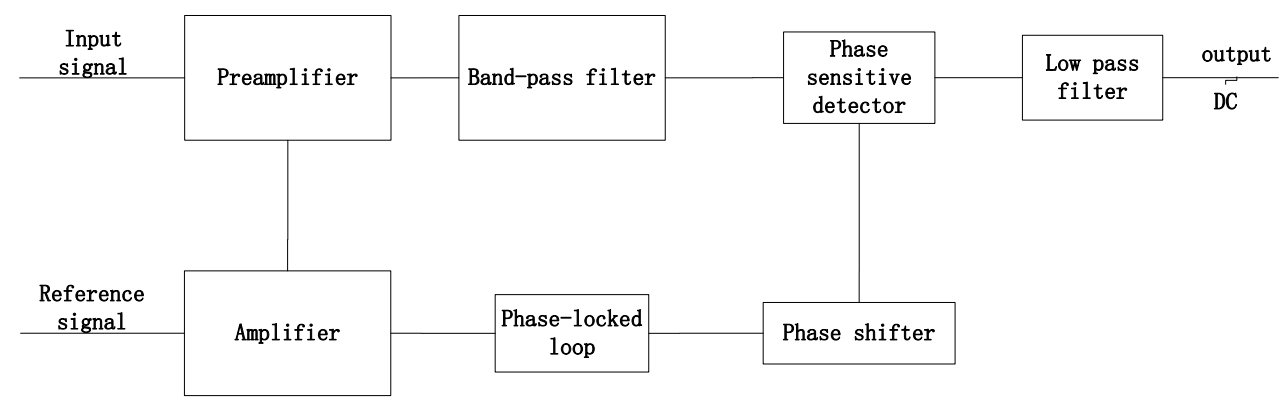

Figure 3. Lock-in Amplifier Block Diagram of the Overall Design

\section{Unit Circuit Design}

\subsection{Low-noise Pre-amplifier}

The main role of the low noise pre-amplifier is to amplify the input signal. It is sufficient to promote the post-stage circuit, and it can make detected process easier.

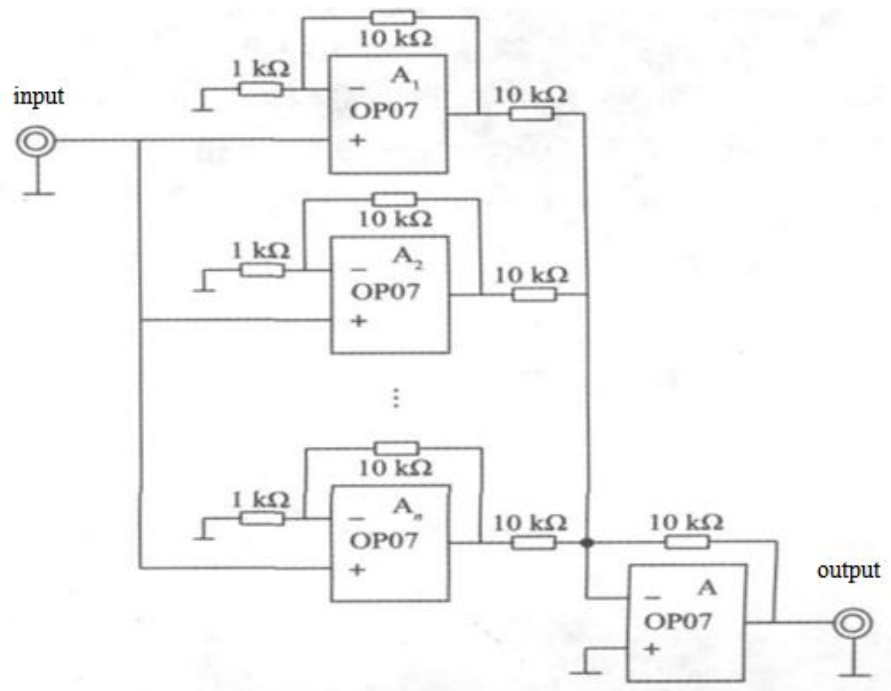

Figure 4. Low-noise Pre-amplifier Circuit Diagrams

The module consists of two parts. The first part is an integrated amplifier and preamplevel parallel input constitutes, and the second part is based on the high-precision instrumentation amplifier AD620. Low-noise preamplifier is designed to suppress the key and match with the front of the detector and the noise. The design itself first from the preamplifier design (including selection of components, based on the processing power on the ground) reduces noise by 10 parallel input, making the amplifier noise reduce times, and the differential mode voltage is about 110 times magnification. The program allows the detector between the front-end pre-amplifier and can be a good convergence to reduce the amplifier noise Figure, reduce noise, and improve signal to noise preamplifier. After class use AD620 gain adjustment can be achieved, it improves the system of common-mode rejection ratio. 


\subsection{Tracking Band-pass Filter}

Tracking band-pass filter can cut away a part of the noise, improve the signal-to-noise ratio, and obtain a better accuracy. Tracking band-pass filter, based on fourth-order bandpass filter, constitutes switched capacitor filter chip LMF100CCN.

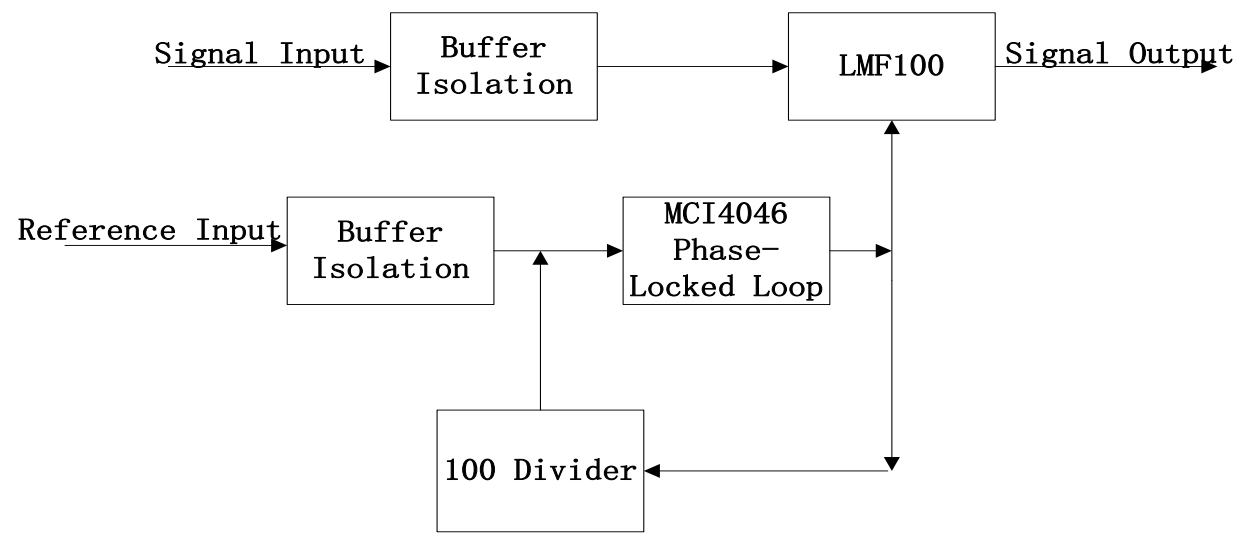

Figure 5. Block Diagram of the Tracking Band-pass Filter

\subsection{Reference Channel Amplifier Design}

Reference channel amplifier has two functions: amplifier and integer the reference signals. Integer circuit is hysteresis comparator which integrates with AD812 high-speed operational amplifier. The reference signal is a square wave integer, amplifying reference signal to the appropriate range. AD812 is a high speed type of integrated operational amplifier which has a high slew rate, allowing the rising and falling edges of the square wave in shorter time. MAX232 is used to convert the RS-232 and TTL level between chips, in order to complete this level conversion, so that the level of post-stage outputs switching signal compatibility.

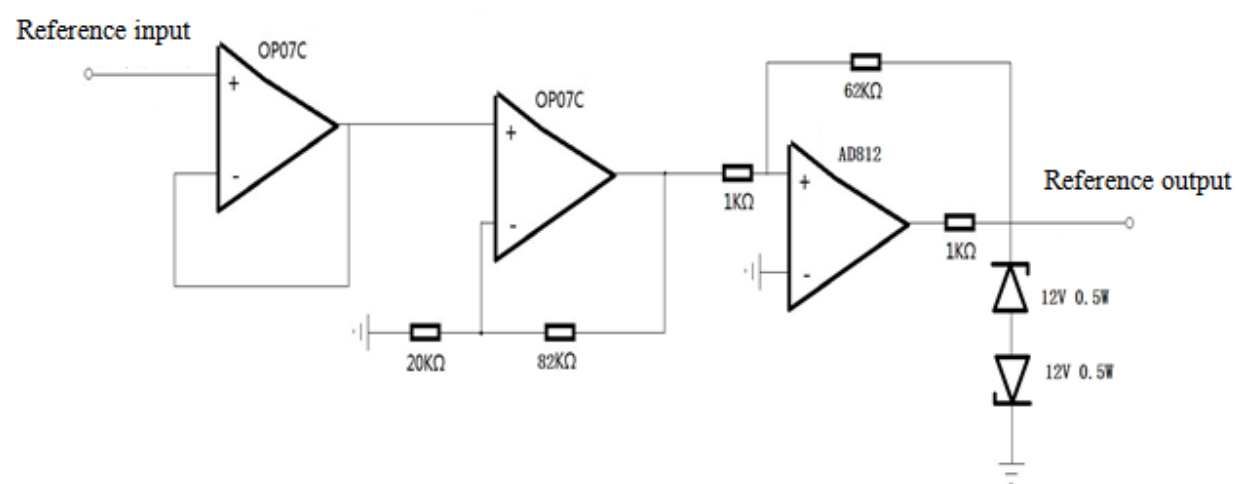

Figure 6. Reference Channel Amplifier Circuit Diagrams

\subsection{PLL Design}

Phase-locked loop (PLL1)can make the amplified reference signal form a square wave with the entire duty cycle of $1: 1$, and then the phase shift is applied to the reference signal and the input signal have the same phase. The circuit diagram of a phase locked loop circuit is shown below.

Phase-locked loop circuit unit based on integrated PLL chip CD4046, aims to become the reference signal integer $50 \%$ duty cycle square wave, like the tracking bandpass filter and phase-sensitive detector of switching signal. 74LS02 and its peripheral circuit form a phase lock indicator circuit [6]. When the reference signal is to meet the requirements, 
the light emits diode D2 emit light; when the reference signal amplitude is too small or the frequency is too high, the PLL loses lock, and D2 extinguishes. The reason for the reference design helps channel monitoring, and analysis of the system does not work properly. TLP521-2 is a non-linear optocoupler. Its role is to isolate the analog and digital ground. Through the optical signal, it is coupled to the switching signal analog circuit, and prevents crosstalk between analog and digital ground. Shown in the following Figure is a schematic diagram of a phase locked loop circuit elements:

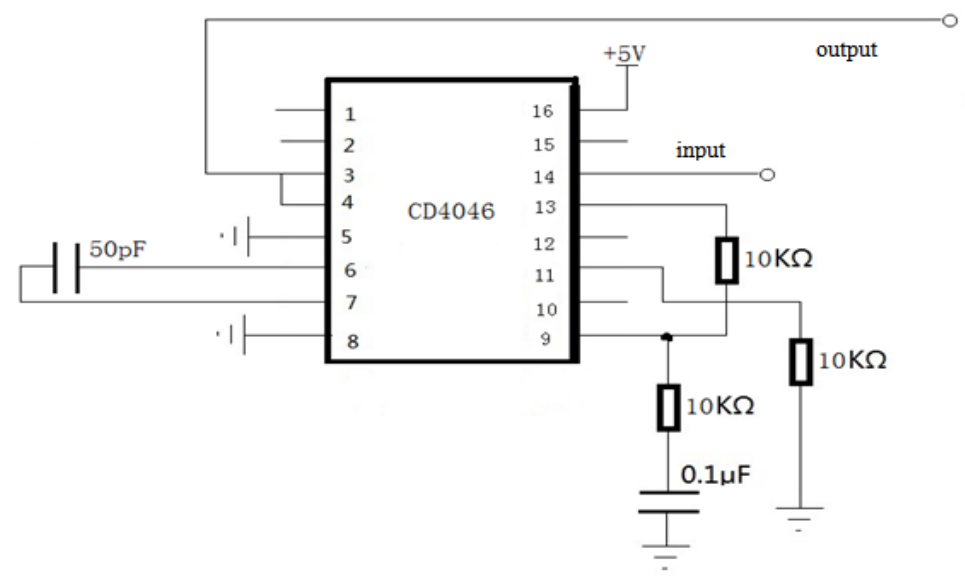

Figure 7. Phase-Locked Loop Circuit Diagrams

\subsection{The Correlator Circuit Design}

The correlator circuit includes the following parts: phase-sensitive detector, low pass filter and the phase shifter. AD630 phase-locked amplifier is mainly composed of the main functions of the comparator, modulator and buffer amplifier. Low pass filter based on low noise integrates operational amplifier OP07 voltage-controlled voltage source of the second-order low-pass filter, with the gain of about 2 times, characteristic of frequency of $0.2 \mathrm{~Hz}$. Phase-shift circuit is also referred to phase difference to produce circuit, analog phase shift circuit frequently uses resistance capacity phase-shift circuit, and the circuit is simple and reliable $[4,5]$.

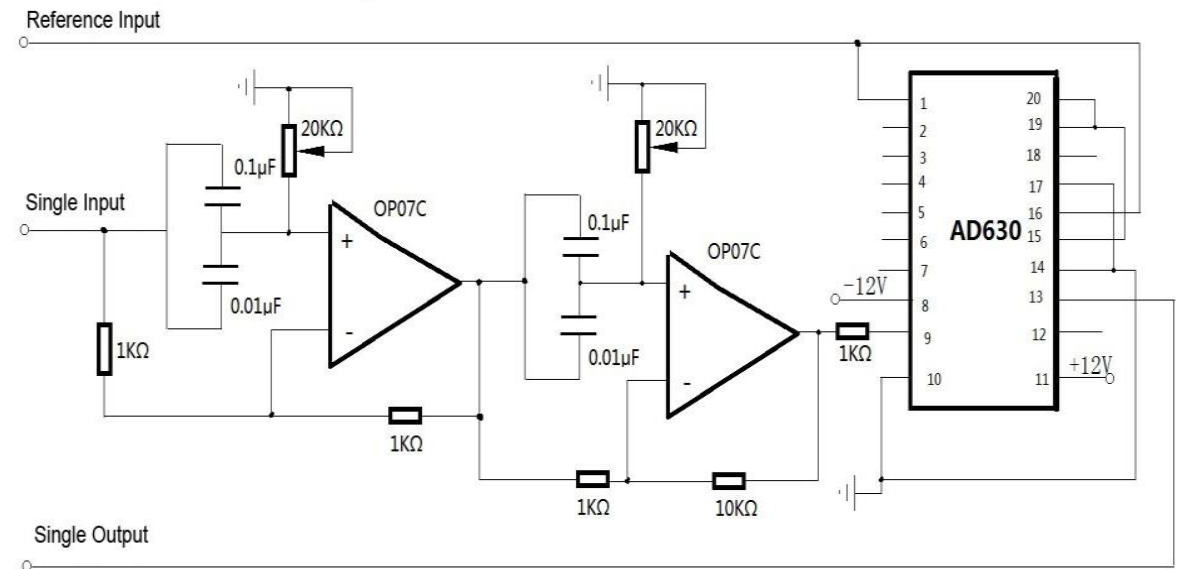

Figure 8. Phase-locked Amplifier Circuit Diagrams

The circuit uses 2 pieces of OP07C high precision integrated op-amp and 1 piece of AD630 composition to make circuit achieve very good effect with precise resistance and 
capacitance 2 pieces of integrated op-amp isolation, phase shift, buffer, the action such as amplification, signal preprocessing of the input, then into AD630, phase-locked amplifier, improve signal-to-noise ratio. In order to improve the stability of the power supply voltage, two capacitors in parallel with the power supply input are used.

The phase-locked amplifier design adopts highly integrated balance AD630 modem chip, which not only simplifies the design, but also effectively suppresses noise and interference. Figures blow are AD630 chip pins and internal structure.

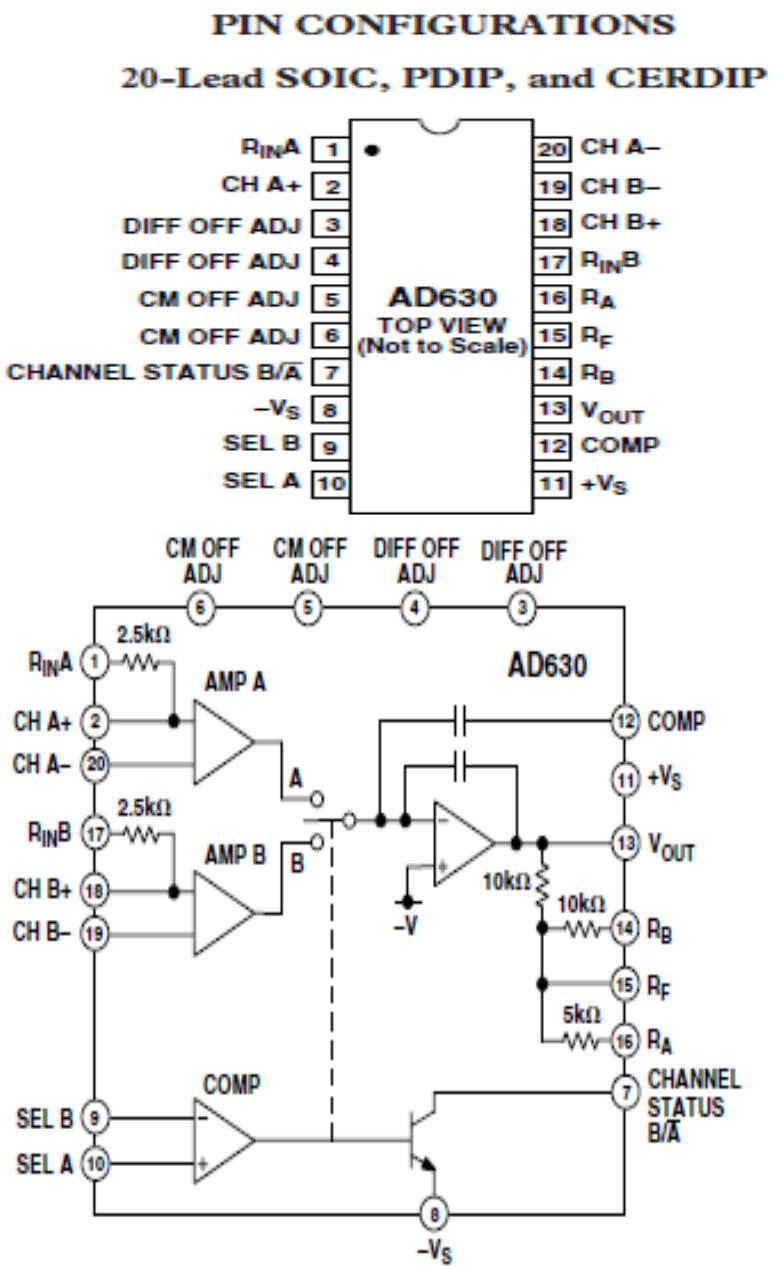

Figure 9. AD630 Pin Internal Circuit Structure Diagram

AD630 is composed of two operational amplifiers, switch A and B switch S, integral amplifier $\mathrm{D}$, comparator output $\mathrm{C}$ (including triode $\mathrm{T}$ ) and the on chip resistor, chip compensation capacitance. Driven by $\mathrm{C}$ switch $\mathrm{S}$ set $\mathrm{A}$ or B. Pin 5, and 6 are commonmode disturbance regulation, pin 3,4 are differential mode disturbance regulation. Channel segregation degree between A and B is more than $100 \mathrm{db}$, time-sharing work. Chip resistor is $\mathrm{SiCr}$ thin film resistor with high stability. Chip compensation capacitor outside the pin connection does not need external capacitance and can perform stable jobs on the closed loop gain. Channel A and B are high precision, high speed integrated operational amplifier. The open-loop voltage gains $A V d \geqslant 100 \mathrm{~dB}$, input offset voltage $\mathrm{Vos} \leqslant 100 \mathrm{uV}$, input offset current Ios $\leqslant 10 \mathrm{nA}$, input bias current IIB $\leqslant 100 \mathrm{nA}$, common mode rejection ratio $\mathrm{KCMR} \geqslant 110 \mathrm{~dB}$, power supply voltage rejection ratio KSVR $\geqslant$ $100 \mathrm{~dB}$, conversion rate of $\mathrm{SR} \geqslant 45 \mathrm{~V} / \mathrm{us}$, or open loop bandwidth produc $\mathrm{B} \omega \mathrm{c} \geqslant 2 \mathrm{MHz}$. 
Chip resistor can form a closed-loop gain of \pm 1 and \pm 2 of symmetric gain amplifier, the precision of the closed-loop gain is higher than $+0.05 \%$, temperature coefficient of 2 $\mathrm{ppm} /{ }^{\circ} \mathrm{C}$.

\subsection{Voltage Detection and Display}

Voltage direct detection and display are used through digital voltage exemplar head ZF5135-DC2V. Resistor divider network R1, R2 is used. Rw is to used to fine-tune the partial pressure ratio to realize the voltmeter full-scale adjustment and show the RMS signal inputs. Shown below is a schematic diagram of the section:

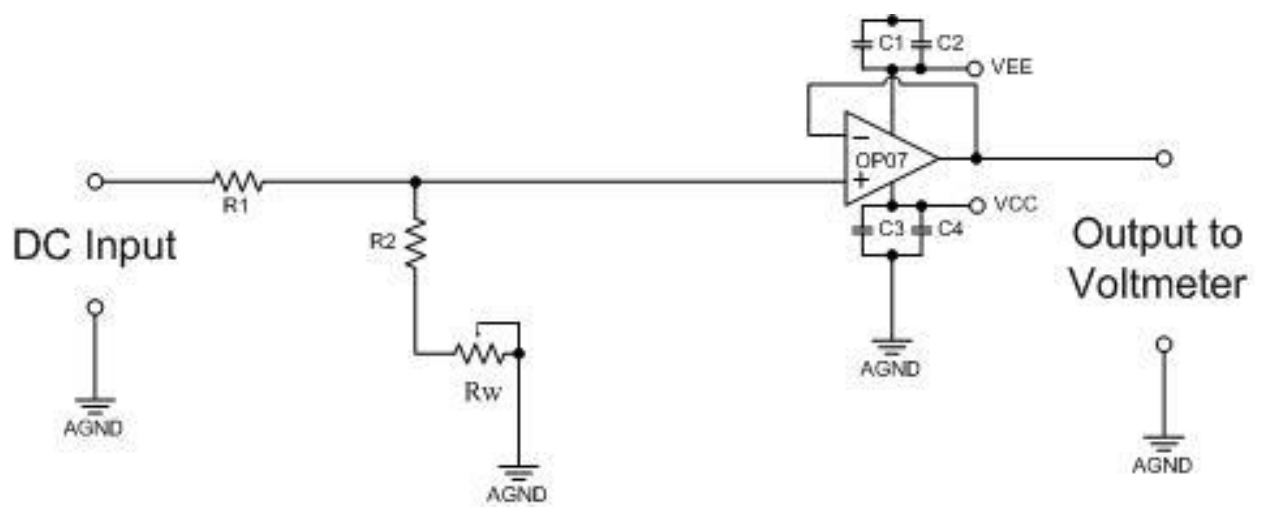

Figure 10. Voltage Direct Detection and Display Diagram

\section{Circuit debugging and Results}

When the signal input is about $400 \mathrm{mV}$, sine wave, the reference signal is approximately $1 \mathrm{KHz} 1 \mathrm{~V}, 1 \mathrm{KHz}$ square wave, the output of this circuit is as follows:

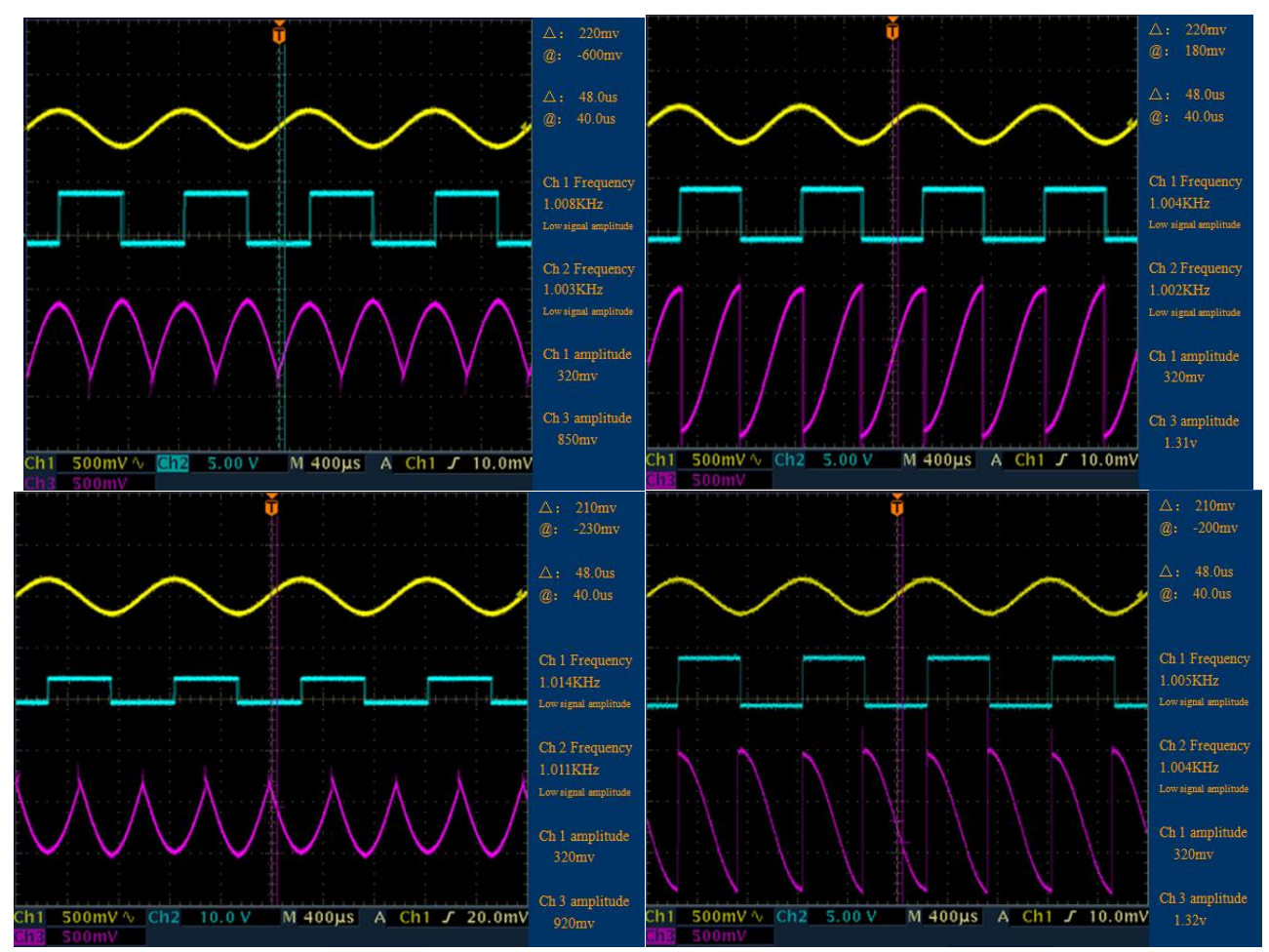

Figure 10. Correlator Output Waveform 
Above the several views, the yellow channel is input waveform signal, the waveform of the reference channel input blue, purple waveform for phase-sensitive detector output.

\section{Conclusion}

According to the above experimental results, the exchange of the circuit is approximately 2 -fold magnification, with the power supply voltage up to $\pm 16 \mathrm{~V}$, the maximum output voltage of $\pm 10 \mathrm{~V}$. Researching AD630 technical manuals, through experimental verification, the upper cut-off frequency at this time is about $1 \mathrm{MHz}$. Under the conditions of $\pm 15 \mathrm{~V}$ supply voltage, the input signal voltage amplitude range is about two $-11 \mathrm{~V}-+14 \mathrm{~V}$. An output signal voltage range is about $\pm 10 \mathrm{~V}$. This frequency and voltage range is capable of meeting most of the weak signal detection. The system can effectively amplify the input signal, achieve the function of extracted the weaker signal from the background noise, and amplify it. Designed for low cost and high performance, this system can make a lot of fruitful work in improving detection accuracy and functional and it is suitable for application in electronics, information science, optics, electromagnetics area. It can precisely measure weak signal from the noise.

\section{References}

[1] G. Jin-Zhan, “The weak signal detection”, Tsinghua university press, (2004).

[2] R. Augulis and D. Zigmantas, "Two-dimensional electronic spectroscopy with double modulation lockin detection: enhancement of sensitivity and noise resistance", Optics Express, vol. 19, no. 14, (2011), pp. 13126-13133.

[3] N. Jia-Sheng, "Used for trace detection of weak signal extraction phase lock amplifying circuit design and implementation".

[4] J. Snoddy, Y. Li, F. Ravet and X. Bao, "Stabilization of electro-optic modulator bias voltage drift using a lock-in amplifier and a proportional integral derivative controller in a distributed Brillouin sensor system", Applied Optics, vol. 14, no. 9, (2007), pp. 1482-1485.

[5] G. Hong, L. Yan and Z. Liyong, "12-electrodes Electrical Capacitance Tomography Detection System Based on Relevant Technology", Journal of Harbin University of Science and Technology, vol. 14, no. 5, (2009), pp. 31-34. 